\title{
FORMAÇÃO E DIAGÊNESE DE ARENITOS DE PRAIA: Uma Revisão Conceitual
}

\author{
FORMATION AND DIAGENÈSE IN BEACHROCKS: \\ A Conceptual Review
}

\author{
Jonatas Malaquias Otavio ${ }^{1}$ \\ jonatasmalaquias@hotmail.com \\ Osvaldo Girão ${ }^{2}$ \\ osgirao@gmail.com \\ Tiago Fernando de Holanda ${ }^{1}$ \\ tiagofholanda@gmail.com \\ Wenderson Sávyo Aguiar da Silva ${ }^{1}$ \\ aguiarsavyo@gmail.com
}

\begin{abstract}
The beach sandstones, or beachrocks, are sediments that underwent a lithification process and are found parallel to the coastline in the intertidal zone, presenting an elongated, narrow shape and cemented by calcium carbonate. The process of diagenesis consists on the formation of the sedimentary rock itself, following deposition, compaction, dissolution, cementation and recrystallization. The study of the formation and diagenesis of beach sandstone is relevant since it permits the understanding of how clastic sedimentary rocks are formed, besides with recognizing how sea level variation behaved during the Holocene, thus allowing the genesis of these features that constitute relevant elements of shelter along coastlines in most tropical environments.
\end{abstract}

Keywords: Beachrocks, sedimentary rock, cementation.

${ }^{1}$ Discente, Departamento de Ciências Geográficas, UFPE.

${ }^{2}$ Docente, Departamento de Ciências Geográficas, UFPE. 


\section{RESUMO}

Os arenitos de praia, ou beachrocks, são sedimentos que sofreram um processo de litifícação e são encontrados paralelamente à linha de costa na zona intermaré, apresentando uma forma alongada, estreita e cimentados por carbonato de cálcio. O processo de diagênese consiste na formação da rocha sedimentar propriamente dita a partir da compactação, dissolução, cimentação e recristalização. $\mathrm{O}$ estudo da formação e diagênese do arenito de praia são relevantes pois possibilita o entendimento de como as rochas sedimentares detríticas são geradas, além de reconhecer como a variação do nível do mar se comportou durante o Holoceno, permitindo a gênese destas feições que se constituem em relevantes elementos de resguardo das linhas de costas em ambientes majoritariamente tropicais.

Palavras-chave: Arenito de praia, rocha sedimentar, cimentação.

\section{CONTEXTO DA PESQUISA}

Os arenitos são importantes para a Arqueologia, devido a sua ampla utilização em construções religiosas e de defesa durante o período colonial no Nordeste do Brasil, além do grande quantitativo de material biogênico presente. Seu papel no ambiente pode servir tanto como um agente dissipativo das ondas na praia, como também como um agente causador de erosão costeira. Os estudos sobre o ambiente permitem uma interpretação da gênese e dos processos a que essa rocha é submetida ao longo do tempo.

Os primeiros relatos sobre esses corpos rochosos no Nordeste foram feitos, segundo Branner (1904), por Pero Vaz de Caminha no início do século XVI. Posteriormente, em 1841, Darwin, em sua passagem pela cidade do Recife-PE, se referiu aos beachrocks como materiais calcíferos com detritos de conchas e também raros seixos, alinhados paralelamente à linha de intermaré. Segundo 
Barreto et al. (2010), Darwin os chamou de "a remarkable bar of sandstone" ou "uma notável barra de arenito". De acordo com Branner (1904 apud FERREIRA JÚNIOR, 2010), os arenitos de praia representam antigas linhas de praia, consolidadas por carbonato de cálcio. Branner em 1904 chamou os arenitos de "the Stone reefs of Brazil" e, na cidade do Recife, iniciou um dos mais famosos trabalhos sobre essa feição fisiográfica (BARRETO et al., 2010) (Figuras 1 e 2).

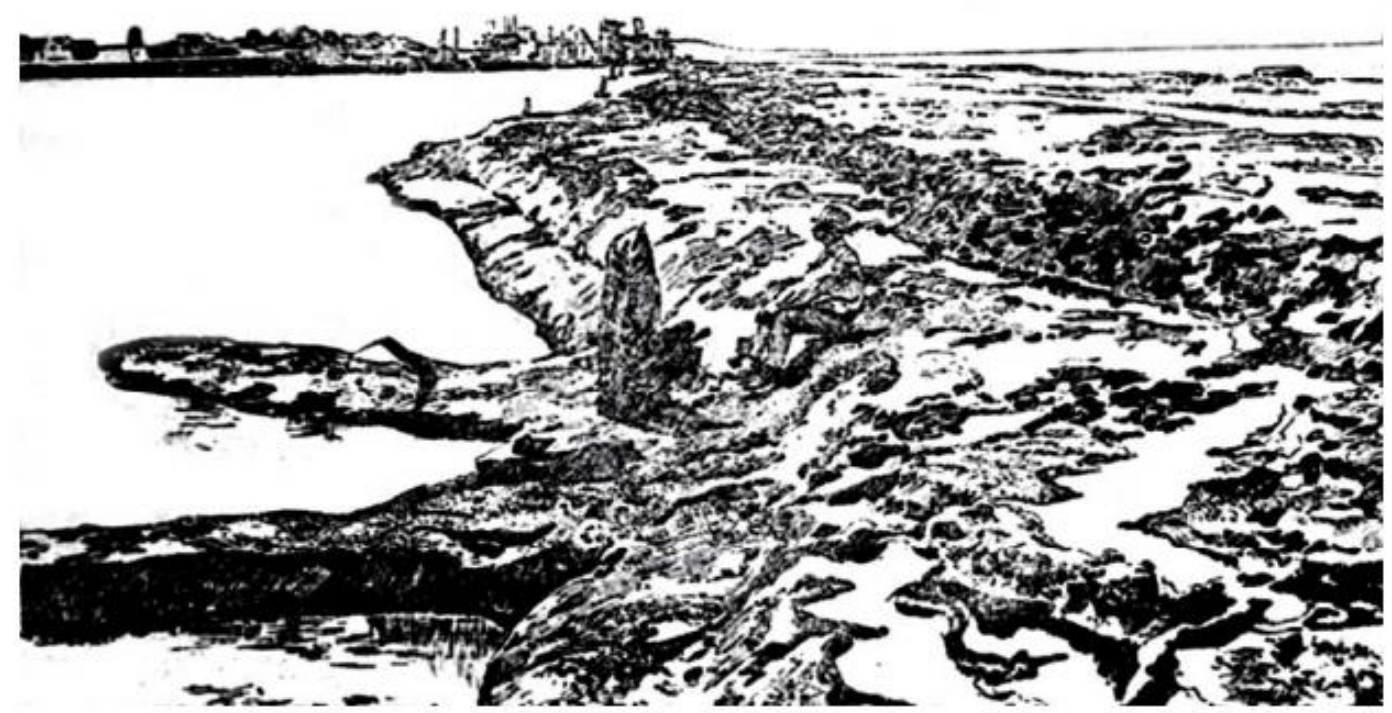

Figura 1: Os recifes naturais do Porto de Pernambuco, segundo ilustração de Branner (1904). Fonte: Barreto et. al. (2010). 


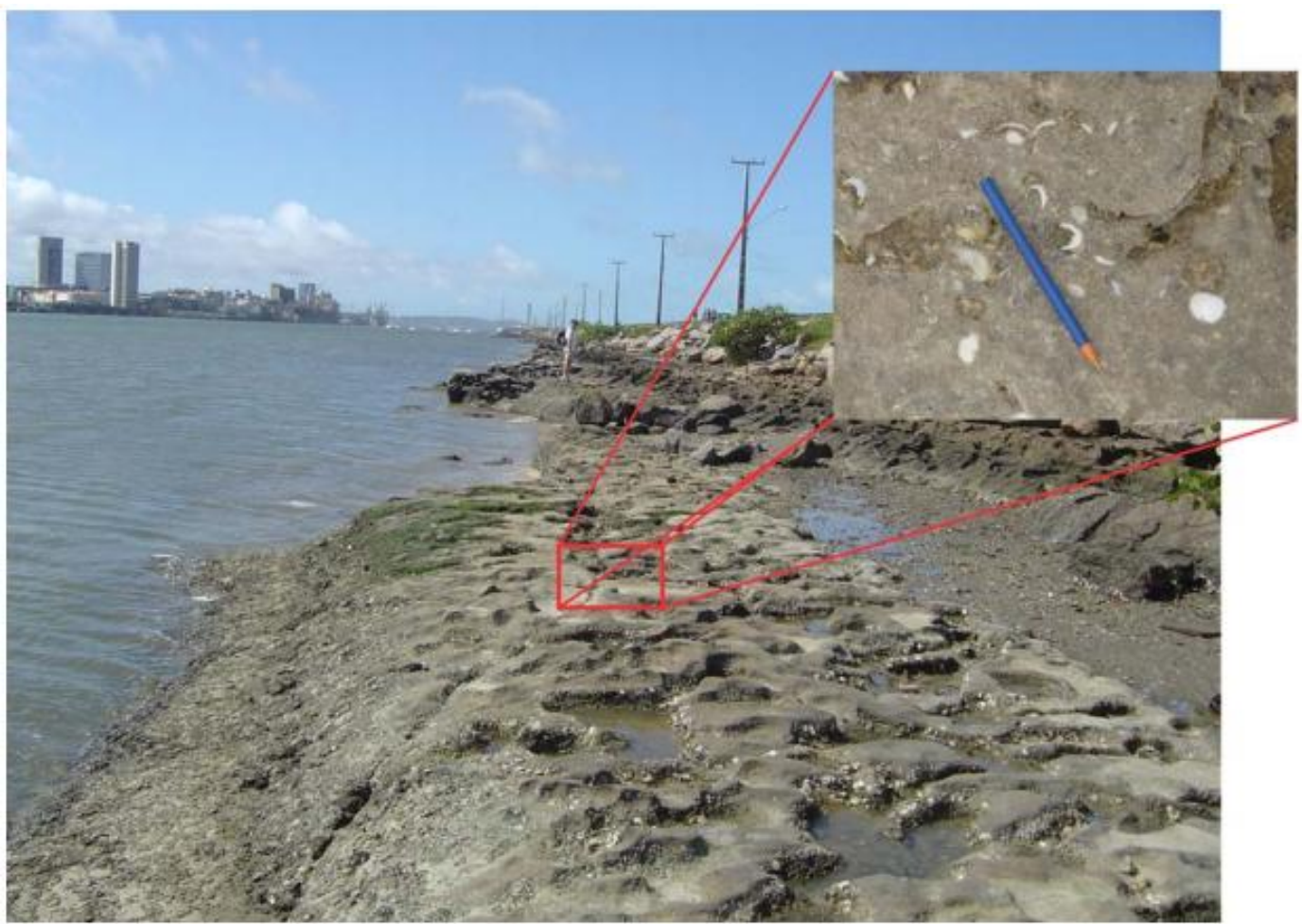

Figura 2: Rocha Praial da Praia do Pina. À esquerda, está a Cidade do Recife e no detalhe, conchas de moluscos. Fotografia obtida 100 anos depois, no local da ilustração de Branner (1904) (Figura1). Fonte: Barreto et. al. (2010).

Como mencionado, os precursores dos estudos sobre arenito de praia na região Nordeste foram Darwin (1841) e Branner (1904). Posteriormente, destacaram-se os trabalhos de Andrade (1955), Van Andel e Laborel (1964), Mabesoone (1964), Morais (1967), Ferreira (1969), Bigarella (1975), Coutinho e Farias (1979), Assis (1990), Oliveira et al. (1990), Caldas (1996), Bezerra et al. (1998), Amaral (2000), Guerra et al. (2005), Bezerra et al. (2005), Ferreira Jr. (2005), Caldas et 
al. (2006), Vieira \& De Ros (2006), Vieira et al. (2007), Barreto et al. (2010), Ferreira Jr. (2010), entre outros.

A terminologia é bastante discutida entre os cientistas. Bigarella (1975) criticou o termo beach rock, sendo que as estruturas sedimentares encontradas são características da antepraia e propôs a terminologia reef sandstone (arenito de recife). Dominguez et al. (1990) afirmam que a terminologia "recife", do ponto de vista sedimentológico, estaria associado a uma construção orgânica. Sendo proposto por eles o termo "bancos de arenito". Suguio (1992 apud FERREIRA JÚNIOR, 2010) sugeriu os termos rocha praial, recife rochoso.

A ocorrência dos arenitos de praias segundo Russel (1962 apud FERREIRA JÚNIOR, 2010), está entre as latitudes de $35^{\circ} \mathrm{N}$ e $35^{\circ} \mathrm{S}$, ou seja, regiões tropicais e subtropicais. Contudo, Kneale \& Viles (2000), Omoto (2001), Rey et al (2004), Vousdoukas et al. (2007 apud FERREIRA JÚNIOR, 2010), apontam a presença de beachrocks em zonas temperadas e frias, mesmo que sejam com formação incipiente como no Japão, Itália, Portugal, entre outros. Devido à ocorrência dos arenitos de praias nestes países, então a ocorrências dessas feições estão restritas entres as latitudes $50^{\circ} \mathrm{N}$ e $50^{\circ} \mathrm{S}$.

Alguns pesquisadores dedicaram estudos a respeito do nível relativo do mar (NRM), onde usam os beachrocks como um indicador geomorfológico e fazendo suas críticas e suas observações a respeito do assunto (Hopley, 1986; Kelletat, 
2006; 2007; Knight, 2007). De acordo com Barreto et al. (2000 apud Barreto et al. 2010), a partir da datação de alguns arenitos de praia no estado do Rio Grande do Norte, foi verificado que a formação dessas rochas vem acontecendo de 7.000 anos até os dias atuais, sendo que as feições que possuem tamanhos maiores de 3 $\mathrm{km}$ possuem idades entre 6.500 e 4.800 anos AP. A utilização de datações absolutas como feitas por Suguio et al. (1985) de conchas de moluscos dessas rochas, permitiram uma maior compreensão do nível do mar no Nordeste. Trabalhos mais recentes como o de Ferreira Jr. (2010), trazem evidências também sobre a variação no nível do mar no Holoceno e a consequente formação de arenitos de praia. Dominguez et al. (1990), afirmaram que os arenitos de praia, encontrados em Pernambuco estariam relacionados com antigos níveis marinhos do Holoceno. Para o arenito de praia se formar ele precisa estar a uma profundidade máxima de 2 metros, o que evidencia um nível marinho mais alto do que o encontrado nos dias atuais.

Os arenitos de praia constituem elementos de proteção para a zona costeira, pois atuam como barreiras naturais impedindo a exposição da costa às ações diretas das ondas, minimizando a possibilidade de processos erosivos nas praias, e também atuando como uma proteção para banhistas.

\section{ÁREA DE ESTUDO}

A título de área-objeto para discussão teórica sobre os arenitos de praia optou-se pela costa de Pernambuco, localizada na região do Nordeste brasileiro, onde na 
zona de intermaré encontram-se as feições conhecidas como arenito de praia. Em algumas praias do Estado de Pernambuco estas rochas são visíveis, como na praia de Boa Viagem, em Recife; Gaibu e Enseada dos Corais, no Cabo de Santo Agostinho, entre outras.

\section{MATERIAIS E MÉTODOS}

A metodologia utilizada consistiu em uma revisão bibliográfica, embasada em artigos científicos, teses, dissertações, livros, capítulos de livros e sites, que continham informações do processo de formação das rochas sedimentares e que descrevam a respeito dos arenitos de praias presentes no Nordeste brasileiro.

\section{RESULTADOS E DISCUSSÕES}

Os arenitos de praia são rochas sedimentares. Os processos de gênese das rochas sedimentares são: meteorização, erosão, transporte, sedimentação e diagênese. Os conceitos desses termos segundo o dicionário geológico-geomorfológico de Guerra et al. (2008) são:

"A meteorização é conjunto de fatores exodinâmicos que intervêm sobre uma rocha acarretando modificações de ordem mecânica-química(...) p.424. A Erosão seria a destruição das saliências ou reentrâncias do relevo, tendendo a um nivelamento ou colmatagem, no caso de litorais, enseadas baías e depressões(...) p.229. O Transporte, fase do trabalho erosivo que segue à ação de destruição realizada pelos agentes exógenos. Numa definição mais ampla, pode-se dizer que o transporte é todo o conjunto de fenômeno geológico que acarreta deslocamento de massa de solo de rochas de um ponto 
a outro(...) p.619. Sedimentação é o processo pelo qual se verifica a deposição dos sedimentos ou de substâncias pelo que poderão vir a ser mineralizadas. Os depósitos sedimentares são resultantes da desagregação ou mesmo da decomposição das rochas primitivas. Esses depósitos podem ser de origem fluvial, pluvial, marinha, glaciária, eólia, lacustre, vulcânica etc(...) p.562. Diagênese é um conjunto de fenômenos que começam a agir modificando os sedimentos desde o início de seu depósito. Este fenômeno, no começo, é unicamente periférico, porém, com o decorrer do tempo, passa a ser mais profundo(...) p.201."

Em outras palavras, meteorização seria o aparato químico que modifica a composição da rocha; a erosão é o conjunto de processos físicos e químicos que possibilitam a remoção dos detritos que tiveram sua mudança na meteorização; transporte seria os movimentos dos detritos que sofreram erosão; a sedimentação o processo que leva os materiais a sofrerem a deposição; e a diagênese seria o último processo para formação das rochas sedimentares, onde os detritos sofrem uma alteração química e física, transformando-se em rochas sedimentares. (GIANNINI, 2003; GUERRA et al., 2008).

Dentro da diagênese existe um conjunto de processos que levam a formação da rocha sedimentar propriamente dita. Os processos são: compactação, cimentação e litificação. Segundo Guerra \& Guerra (2008, p.150-151). "compactação é a diminuição da espessura do pacote de sedimentos provocada pelo aumento da carga e possibilitada pela existência de porosidade nos sedimentos". A compactação pode ter dois processos: químicos e mecânicos. $\mathrm{O}$ processo químico acontece devido à pressão que os detritos sofrem com a dissolução dos minerais. 
Já no processo mecânico não existe um processo químico e sim físico, com a quebra e deformação dos grãos com o empacotamento intergranular (GIANNINI, 2003).

De acordo com Grotzinger e Jordan (2013, p. 135), a Cimentação é “o processo onde os minerais dissolvidos podem precipitar nos poros entre as partículas sedimentares e uni-las". A natureza do cimento pode ser silicosa, argilosa, calcária ou ferruginosa (Guerra e Guerra, 2008). A cimentação ocorre a partir da cristalização dos sedimentos pelos íons dissolvidos nas soluções intersticiais. (GIANNINI, 2003).

O processo de cimentação nos arenitos de praia estaria relacionado à precipitação de carbonatos da água do mar, a partir da evaporação da água intersticial por processos físico-químicos ou por crescimento microbiológico que produzem calcita magnesiana. O tipo de cimento que existe em cada arenito dependerá das características químicas e físicas das águas a partir das quais ele foi precipitado. A calcita, que é um dos principais tipos de cimento, é normalmente precipitada a partir da água doce ou marinha, enquanto que a aragonita é comumente precipitada a partir de soluções de alto potencial iônico, como a água do mar (COUTINHO e FARIAS, 1979; MOORE, 1973; KOMAR (1976) apud FERREIRA JÚNIOR, 2011). Segundo Ferreira Júnior et al. (2011), além de arenitos cimentados por carbonato de cálcio, na costa nordestina é comum 
encontrar arenitos cimentados por óxido de ferro, que são associados à Formação Barreiras.

De acordo com Ferreira Jr. (2010, p.547) "Vários estudos relativos ao tipo de composição do cimento dos arenitos de praia apontam que não existe um único processo na sua formação". O referido autor afirma que, o que as teorias possuem em comum é com relação ao lugar de formação, que segundo ele, ocorre na zona de estirâncio e com isso pode se diferenciar de outros tipos de depósitos em função dessa característica.

Até o momento não foi identificado por estudos se o que forma o arenito de praia é apenas um fator ou se existem vários fatores envolvidos. O que de fato já se atestou foi que ele tem uma rápida formação o que o diferencia de outras rochas, inclusive sedimentares.

Friedman (1998) comprovou que a cimentação em ambiente praial pode ocorrer em um tempo extremamente curto. Tal comprovação ocorreu mediante um experimento realizado nas Bahamas, onde uma lata de sardinha acumulou ao longo de um ano um total de $383 \mathrm{~g}$ de material carbonático cimentante.

Os materiais que os arenitos de praia apresentam, bem como o tipo de cimento que ele possui, podem variar de acordo com o local onde esse arenito é encontrado. Por exemplo, no estudo realizado por Alexanderson (1972), no Mar 
Mediterrâneo, o arenito é formado por quartzo, feldspatos, bioclastos e cimentado por calcita magnesiana. No Togo (África) em trabalho de Amieux et al. (1989) os arenitos são formados por quartzo, feldspatos, minerais pesados e bioclastos e cimentados por calcita magnesiana métrica. Já nas Ilhas Canárias (Espanha) Calvet et al. (2003) constatou que os arenitos de lá são formados por quartzo, grãos vulcânicos e cimentados por aragonita fibrosa e calcita altamente magnesiana. Os arenitos do Mar Egeu são formados segundo Ergin et al (2008) por quartzo, feldspatos, biotita e minerais opacos. Os de Belize (América Central) de acordo com Gischler e Lomando (1997) são formados por quartzo, oóides, microrganismos e cimentados por Aragonita, calcita altamente magnesiana e cimentos do tipo meniscos.

Os trabalhos citados demonstram como o material que pode formar os arenitos, bem como a sua cimentação, são variáveis de acordo com o material disponível no momento de sua formação. De acordo Ferreira Jr. et al. (2005) os principais tipos de cimentos observados na literatura são: aragonita micrítica, aragonita acicular, calcita magnesiana micrítica, calcita magnesiana peloidal e calcita magnesiana paliçada.

Saindo dos processos genéticos para uma compreensão a respeito variação do nível do mar a partir do Holoceno, alguns estudos tiveram muitas contribuições. Alguns indicadores são utilizados para a obtenção do resultado do nível médio do mar a partir do Holoceno (Figura 3). Entre eles as conchas incrustadas nos 
beachrocks, vermetídeos, sambaquis e fragmentos de madeira, podem ser submetidos à datação pelo método Carbono 14 .

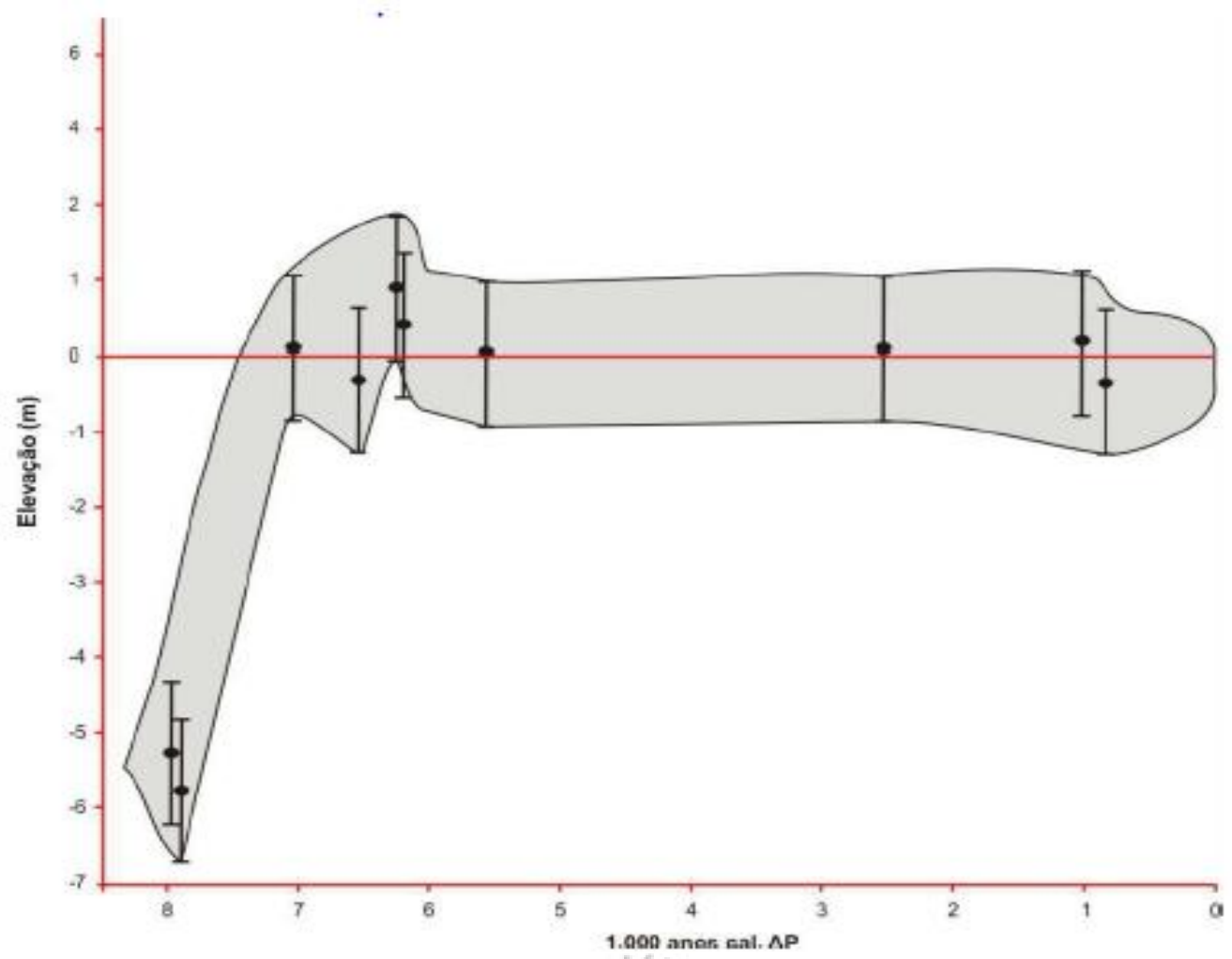

Figura 3: Curva de elevação do nível do mar no Holoceno para costa de Pernambuco, a qual representa o nível mínimo e máximo do nível médio das marés, (FERREIRA JUNIOR, 2010).

Em Pernambuco, Van Andel e Laborel (1964 apud FERREIRA JÚNIOR, 2010) e Delibrias e Laborel (1971 apud FERREIRA JÚNIOR, 2010), com base na datação radiocarbono, iniciaram a reconstrução do nível do mar no Holoceno através dos 
indicadores citados, sendo encontrados materiais que teriam idades entre 5.900 anos A.P. a 1.119 anos A.P.

A Figura 4 apresenta três imagens da costa do município do Cabo de Santo Agostinho, sendo que as imagens "A" e "B" são de ortofotocartas cedidas pela CONDEPE/FIDEM, onde a imagem "A" é do ano de 1989, a imagem "B" do ano de 1975. Essas duas imagens mostram a praia que se encontra diretamente exposta à ação das ondas, devido a ausência dos arenitos de praias naquela porção. Esta feição acarreta na formação de uma pequena enseada.

No local da Figura 4 existe uma parede de concreto feita na parte da pós-praia que foi colocada para conter a erosão localizada nesta pequena enseada. Esta construção foi feita pela prefeitura do município, que nos últimos 10 anos, vem demonstrando uma preocupação em relação à erosão da praia, justamente devido à extensiva ocupação urbana presente sobre a linha de costa. A imagem "C" é do ano de 2016, comparando as imagens "A, B e C" observa-se que na imagem "C" a pequena enseada sofreu um aumento de tamanho desde 1975 até 2016.

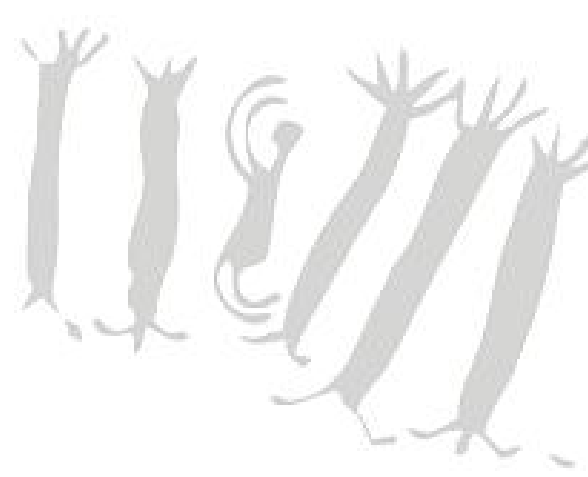




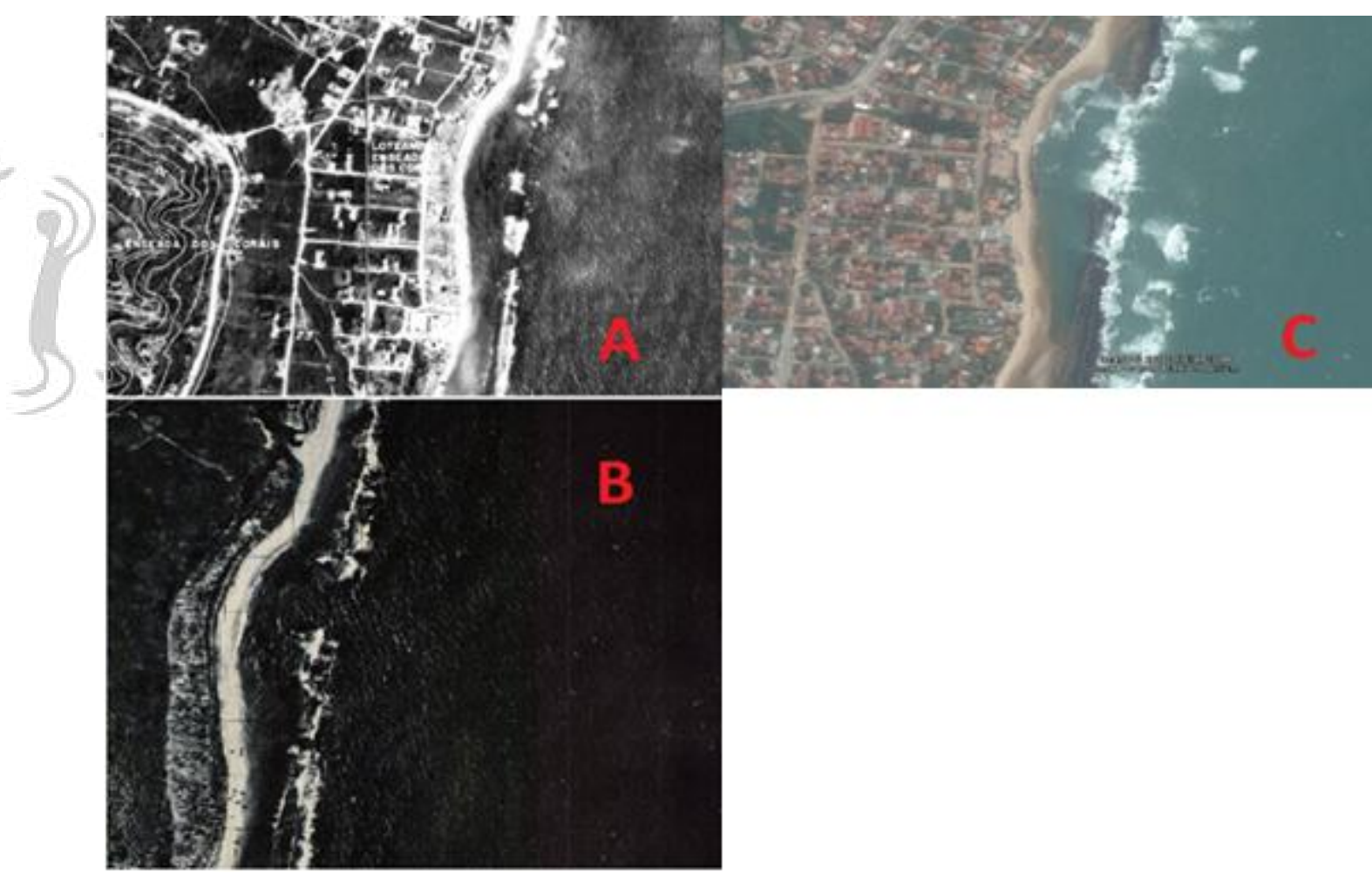

Figura 4: Imagens da Praia de Enseadas dos Corais no município do Cabo de Santo Agostinho, PE. Ortofotocartas (A, B) de 1989 e 1975 respectivamente, disponibilizadas pela CONDEPE/FIDEM, imagens (C) Google Earth de 2016.

\section{CONSIDERAÇÕES FINAIS}

Os estudos da formação e diagênese do arenito de praia é relevante, pois, a partir destes, pode-se observar como as rochas sedimentares, de uma forma geral, se formam no contexto transicional continente-mar. Outro aspecto relevante destes estudos é a possibilidade de entendimento da influência que a variação do nível do mar possui no processo de morfogênese destas feições durante o Holoceno, visto 
que para sua formação é necessária certa profundidade. Sendo assim, é possível relacionar sua gênese a um nível relativo do mar mais elevado do que o atual.

Ademais, por sua estrutura resistente, os beachrocks comportam-se como feições protetoras da linha de costa, promovendo um resguardo daquela em relação a processos erosivos que podem causar prejuízos estruturais. $\mathrm{O}$ reconhecimento da dinâmica destas feições objetiva minimizar possíveis destruições/usos danosos à morfodinâmica praial.

\section{REFERÊNCIAS BIBLIOGRÁFICAS}

ALEXANDERSSON, T. Intergranular growth of marine aragonite and $\mathrm{Mg}$ calcite: evidence of precipitation from supersaturated sea-water. Journal of Sedimentary Petrology, v. 42, p. 441-460, 1972.

AMARAL, R.F. Contribuição ao estudo da evolução morfodinâmica do litoral oriental sul do Rio Grande do Norte, entre Ponta de Búzios e Baia Formosa. Tese de doutorado. Curso de Pós-Graduação em Geociências. UFRS. Porto Alegre. 2000.

AMIEUX, P., BERNIER, P., DALLONGEVILLE, R., MEDWICK, V., 1989. Cathodoluminescence of carbonate-cemented Holocene beachrock from the Togo coastline (West Africa): an approach to early diagenesis. Sedimentary Geology, 65: 261272 .

ANDRADE, G.O. Itamaracá: contribuição para o estudo geomorfológico costa pernambucana. Ofic. Est. Pernambuco, Recife. 1955.

ASSIS,H.M.B. Estudo dos Beach Rocks do Litoral sul de Pernambuco com base em evidências petrográficas e isotópicas. Dissertação de Mestrado, Departamento de Geologia, Centro de Tecnologia e Geociências da Universidade Federal de Pernambuco, Recife. 1990. 
BARRETO,A.M.F.; ASSIS,H.M.B.; BEZERRA,F.H.R.; SUGUIO,K. Arrecifes, a Calçada do Mar de Recife, PE - Importante registro holocênico de nível relativo do mar acima do atual. In: Winge,M.; Schobbenhaus,C.; Souza,C.R.G.; Fernandes,A.C.S.; Berbert-Born,M.; Sallun filho,W.; Queiroz,E.T. (Edit.) Sítios Geológicos e Paleontológicos do Brasil. SIGEP. 2010.

BEZERRA, F.H.R., LIMA-FILHO, F.P.; AMARAL, R.F.; CALDAS, L.H.O.; COSTANETO, L.X. Holocene coastal tectonics in NE Brazil. In: Stewart, I. S., Vita-Finzi, C. Coastal Tectonics. Geological Society, Special Publications, v. 146, p. 279-293. 1998.

BEZERRA, F.H.R.; AMARAL, R.F.; LIMA-FILHO, F.P.; FERREIRA JR. A.V.; SENA, E.S.; DINIZ, R. F. Beachrock Fracturing in Brazil. Journal of Coastal Research, v. 42, p. 319- 332. 2005.

BIGARELLA, J.J. Reef sandstones from Northeastern Brazil (a survey on sedimentary structures). In: Simpósio Internacional sobre o Quaternário, 1, Academia Brasileira de Ciências, Anais, p. 395-410. 1975.

BRANNER J.C. 1904. The stone reefs of Brazil, their geological and geographical relations with a chapter of the coral reefs. Harvard Coll. Mus. Comp. Zool. Bull, 44: 1285.

CALDAS L.H.O. Geologia Costeira da Região de São Bento do Norte e Caiçara, Litoral Potiguar. Departamento de Geologia, Universidade Federal do Rio Grande do Norte, Natal, Relatório de Graduação. 1996.

CALDAS, L.H.O.; STATTEGGER, K.; VITAL, H. Holocene sea-level history: evidence from coastal sediments of the northern Rio Grande do Norte coast, NE Brazil. Marine Geology, v. 228, p. 39-53. 2006.

CALVET, F., CABRERA, M. C., CARRACEDO, J. C., MANGAS, J., PEREZTORRADO, F.J., RECIO, C., TRAVE, A., 2003. Beachrocks from the island of La Palma (Canary Islands, Spain). Marine Geology, 197:75-93.

COUTINHO, P. N.; FARIAS, C. C. Contribuição à origem dos recifes do Nordeste. In.: Simpósio de Geologia do Nordeste, 9, Anais, p. 236-240. 1979. 
DARWIN, C. On a remarkable bar of sandstone of Pernambuco on the coast of Brazil. London: Magazin \& Journal Sciences, p. 257-261, 1841.

DOMingueZ, J. M. L; BITTENCOURT, A. C. S. P; LEÃO, Z. M. A. N; AZEVEDO, A. E. G. Geologia do quaternário costeiro do estado de Pernambuco. Revista Brasileira de Geociências, Volume 20, 1990.

FERREIRA JUNIOR, A. V. Mapeamento da zona costeira protegida por arenito de praia (beachrocks) em Nísia Floresta - RN. 2005. 86 p. Dissertação (Mestrado em Geodinâmica e Geofísica) - Universidade Federal do Rio Grande do Norte. Natal. 2005.

FERREIRA JUNIOR, A. V. Mapeamento e Estudo Petrológico de arenitos de praia (beachrocks): evidências da variação no nível do mar no Holoceno, na costa central de Pernambuco, Ano de obtenção: 2010.

FERREIRA JUNIOR, A. V.; ARAÚJO, T. C. M.; COLTRINARI, L. Ambientes de Formação, Processos de Cimentação de Arenitos de Praia e Indicadores de Variações do Nível do Mar. Revista Brasileira de Geografia Física, 5, p938-960. 2011.

FERREIRA, Y.A. Recifes de arenito de Salvador, Bahia. Anais da Academia Brasileira de Ciências, v. 41, p. 542-548. 1969.

FRIEDMAN, G.M. Rapidity of marine carbonate cementation - implications for carbonate diagenesis and sequence stratigraphy: perspective. Sedimentary Geology, Elsevier. v. 119, p. 1-4, 1998.

GIANNINI, P. C. F. Depósitos e rochas sedimentares. In: TEIXEIRA, W.; TOLEDO, C. M.; FAIRCHILD, T. R.; TAIOLI, F. (Org.). Decifrando a Terra. São Paulo: oficina de textos, 2003. p. 286-304.

GISCHLER, E., LEMANDO, A. J. Holocene cemented beach deposits in Belize, Sedimentary Geology, 110: 277-297 1997.

GROTZINGER, J.; JORDAN, T. Para Entender a Terra. $6^{\text {a }}$ Edição Porto Alegre: Bookman. 2013.

GUERRA, A. J. T.; GUERRA, A. T.. Novo dicionário geológico-geomorfológico. $6^{\circ}$ edição. Rio de Janeiro: Bertrand Brasil, 2008. 652p. 
GUERRA, N.C.; KIANG, C.F.; SIAL, A.N. Carbonate cements in contemporaneous beachrocks, Jaguaribe beach, Itamaracá island, northeastern Brazil: petrographic, geochemical and isotopic aspects. Anais da Academia Brasileira de Ciências, v. 77(2), p. 343-352. 2005.

HOPLEY, D. Beachrock as a sea-level indicator. In: PLASSCHE, O. (Ed.) Sea level research: A manual for collection and evaluation of data. Geo books, Norwich, pp. 157173. 1986.

KELLETAT,D. Beachrock as Sea-Level Indicator? Remarks from a Geomorphological Point of View, Journal of Coastal Research, 22 (6), 1558-1564. 2006.

KELLETAT,D. Reply to: Knight, J. 2007. Beachrock Reconsidered. Discussion of: Kelletat, D., 2006. Beachrock as Sea-Level Indicator? Remarks from a Geomorphological Point of View, Journal of Coastal Research, 22 (6), 1558-1564; Journal of Coastal Research, 24(4), 1074-1078. Journal of Coastal Research. 23 (6 ) 1605-1606. 2007.

KNIGHT,J. Beachrock Reconsidered. Discussion of: Kelletat, D. 2006. Beachrock as Sea-Level Indicator? Remarks from a Geomorfological Point of View. Journal of Coast Research 23 (4) 1074-1078. 2007.

MABESOONE, J.M. Origin and age of the sandstone reefs of Pernambuco (Northeastern Brazil). Journal Sedimentary Petrology, v. 34, p. 715-726. 1964.

MORAIS, J. O. Contribuição ao estudo dos "Beachrocks" do nordeste do Brasil. Laboratório de Ciências do Mar da Universidade Federal do Ceará. Trabalhos Oceanográficos, Univ. Fed. Pernambuco, v. 9, p. 79-94. 1967.

OLIVEIRA, M.I.M.; BAGNOLI, E.; FARIAS, C.C.; NOGUEIRA, A.M.B.; SANTIAGO, M. Considerações sobre a geometria, petrografia, sedimentologia, diagênese e idade dos "beachrocks" do Rio Grande do Norte. $36^{\circ}$ Congresso Brasileiro de Geologia, Natal-RN, 2: 621-634. 1990.

\section{SUGUIO,K.; $\quad$ MARTIN,L.; $\quad$ BITTENCOURT,A.C.S.P.; $\quad$ DOMINGUEZ,J.M.L.;} FLexor,J.M.; AZEVEDO,A.E.G. Flutuações do nível relativo do mar durante o Quaternário Superior ao longo do litoral brasileiro e suas implicações na sedimentação costeira. Revista Brasileira de Geociências. São Paulo. 15 (4): 273-286. 1985. 
VAN ANDEL, T. H.; LABOREL, J. Recent high relative sea level stand near Recife, Brasil. Science, v. 145, p. 580 -581. 1964.

Vieira, M.M.; De Ros, L.F. Cementation patterns and genetic implications of Holocene beachrocks from northeastern Brazil. Sedimentary Geology, v. 192, p. 207- 230. 2006.

VIEIRA, M.M.; DE ROS, L.M.; BEZERRA, F.H.R. Lithofaciology and palaeoenvironmental analysis of Holocene beachrocks in northeastern Brazil. Journal of Coastal Research, v. 23, p. 1535-1548. 2007. 